\title{
Acciones y estrategias para un Programa de Tenencia Responsable de Animales en Chile
}

\author{
Claudia Aguirre
}

Universidad de Chile

\section{Resumen}

La Tenencia Responsable de Animales (TRA) no constituye un concepto global y único; en Chile sus principios rectores descansan en un proyecto de ley y actualmente su regulación se sustenta en aportes de diversos reglamentos, leyes y ordenanzas municipales; sin una definición conceptual y operativa de indicadores. La no existencia de una política pública para abordar el origen de la problemática de tenencia animal, repercute en la salud de personas y animales; y del ambiente. El objetivo del estudio fue identificar acciones y estrategias componentes de un Programa de TRA para ser implementado en Chile, combinando una Revisión Sistemática de literatura científica y gris para identificar contenidos pertinentes y una metodología Delphi dirigida a 15 actores claves nacionales para valorar los contenidos. En la revisión de literatura se identificaron seis grandes acciones y cincuenta estrategias para abordar la TRA. Todas las acciones fueron valoradas por los expertos, poniendo mayor énfasis en estrategias de "control de población animal", "legislación" y "educación". Otras estrategias como "eutanasia" y "refugios" generaron controversias y no existió acuerdo para que sean incorporadas. Se definió que las municipalidades son la entidad que tiene el rol de la implementación del Programa.

Palabras clave: Tenencia de animales, control de población animal, bienestar animal.

Actions and strategies for a Responsible Pet Ownership Program in Chile

\begin{abstract}
The Responsible Pet Ownership (RPO) at present, does is not a global concept and one; in Chile its guiding principles is in a draft law, actually the practices of regulation are in laws, regulations, municipal ordinances, without a conceptual definition and operational indicators. The abscense of a public policy about pet ownership problem, has repercussions in the health of people, animal care and the environment. The objetive of the study was identified in the actions and strategies of a RPO program to be implemented in Chile, combining a Systematic review of cientific and not-cientific literature and a Delphi method addressed to fifteen national social actors, to value contents. Six global actions and fifty strategies were identified in a Systematic review as relevant to approach a RPO. All actions were valued by experts, with greater emphasis on "animal population control", "legislation" and "education" strategies. Other strategies such as euthanasia and shelters generated controversy and there was no agreement to incorporate them. It was defined that the municipalities are the entity that has the role of the implementation of the Program.
\end{abstract}

Keywords: Pet ownership, population control animal, animal welfare.

*Dirección de correspondencia [Correspondence

address]: Claudia Aguirre, Universidad de Chile

E-mail: claudiaguirre@gmail.com 


\section{Introducción}

\section{Tenencia Responsable de animales}

La Tenencia Responsable de Animales (TRA) no constituye un concepto global y único que la defina, sin embargo, hace referencia a las condiciones y obligaciones que deben ser adoptadas por los propietarios de un animal para asegurar el bienestar de su mascota. El déficit de marcos conceptuales que permitan promover la TRA repercute en una serie de problemáticas públicas, que afectan la salud ambiental y que impactan en la población humana y animal (Soto, 2013). En encuestas ministeriales de consulta ciudadana se ha relevado el tema de la presencia de "perros vagos" en las vías públicas, definido como una problemática con consecuencias para la salud humana, ambiental y urbana en relación a otros temas, ocupando el primer lugar en la consulta de salud y vivienda $(50,4 \%$ y $63 \%$ de los encuestados respectivamente); y tercer lugar en la de medio ambiente $(7,4 \%$ de los encuestados) (Ministerio de Salud, 2006, Ministerio de Vivienda y Urbanismo, 2015, Ministerio de Medio Ambiente, 2017).

La evolución en la relación hombre-animal, particularmente en la sociedad occidental, se vincula a procesos de domesticación en los cuales el recurso animal se conceptualiza como un "bien" o "servicio", o bien como proveedor de protección y compañía para el ser humano (Fuentes Cintra et al., 2006). El término "animal de compañía" ha sido adoptado por algunos investigadores y profesionales para referirse directamente al principal rol que se cree que cumplen estos animales, también se utiliza el término "mascota" para referirse a una vasta gama de animales mantenidos domésticamente.

Según la World Animal Protection-WPA (ex World Society For The Protection Of AnimalsWSPA), ambos conceptos reúnen elementos comunes, hacen referencia al animal doméstico (perros, gatos y algunas aves), al cuidado personal por parte del propietario, a la contribución que hacen a la vida diaria del ser humano y del grupo familiar y a la oferta de beneficios no comerciales, pese a tener un valor económico intrínseco (Main, 2007).

\section{Bienestar Animal para la TRA}

Los temas que trata el bienestar animal se han consolidado, abarcando tres ámbitos: ciencia, ética y ley (Broom, 2011), entendiéndose como una responsabilidad ética sobre los animales que están bajo el cuidado y manejo de las personas (Muldoon et al., 2009).

La Organización Mundial de Sanidad Animal (Organización Mundial de Sanidad Animal, 2016), menciona que deben cumplirse cinco libertades para conseguir un estado de bienestar para un animal: 1) alimentación y dieta adecuada; 2) vivir en un ambiente adecuado; 3 ) estar protegido contra el dolor, lesiones, el sufrimiento y la enfermedad; 4) libertad para expresar el comportamiento natural, con espacio e instalaciones adecuados, con compañía de animales de la propia especie u otros; 5) libertad de sufrir miedo ni angustia, proveer condiciones y trato que eviten el sufrimiento psíquico.

Son esencialmente relevantes las políticas ideadas por la OIE, debido a que muchos de los países que no disponen de legislación específica sobre bienestar animal aplican los lineamientos de esta organización como normas. Sin embargo, cabe destacar que entregarle relevancia o preocupación al bienestar animal está intrínsecamente relacionado con la ética, de modo que la toma de decisiones de políticas públicas de TRA en este ámbito no sólo dependerá del conocimiento que se genere sobre el sufrimiento de las mascotas bajo ciertas condiciones, generadas en el contexto humano, sino en valores superiores a los cuales adscribe un determinado país (G.2, 2012).

\section{Legislación y estrategias en TRA}

La legislación en protección animal demuestra las preocupaciones éticas de una sociedad. Los lineamientos y recomendaciones que generan los organismos internacionales tienen influencia en el plano legislativo, existen especificaciones que abordan directa o indirectamente la TRA, que van desde lo punitivo o castigo hacia las personas, hasta la prevención de conductas de relación inadecuada con los animales y el medio ambiente.

Las diferencias en los marcos regulatorios se evidencian entre y dentro de los países. La recopilación de estrategias implementadas para el control de población de perros realizada por la OIE en los países que son miembros (172), muestra que los países desarrollados cuentan con planes o programas implementados y estrategias claras, no así los países subdesarrollados. Se identificó que países más pobres, persisten en implementar sistemas de eliminación masivos con métodos crueles, mientras que en los países más ricos, se emplean procedimientos de control acordes al bienestar animal como la identificación y registro de animales (Dalla Villa et al., 2010).

Particularmente en Suecia, Estados Unidos y Australia, se reconocen algunos beneficios y la im- 
portancia de la relación con animales (Gómez et al., 2007). Junto con EEUU, los países de la Unión Europea poseen leyes de protección y bienestar animales de larga data.

Países latinoamericanos como Colombia (Gómez et al., 2007), Perú (Ministerio de Salud de Perú, 2003; Ley 26.842, 1997), Argentina (Ley 14.346, 1954; Ley 13.879, 2008) y Uruguay (Ley 18.471, 2009) centran sus políticas de TRA en la protección animal principalmente de animales de compañía, estableciendo obligaciones para los propietarios, medidas sanitarias y de tenencia.

En definitiva, es posible extraer a partir de la revisión de legislación de países que existen distintas vías de acción para desarrollar la TRA, por medio de leyes, programas y normas que delimitan el accionar de las personas y mejoran la relación hombre-animal. Sin embargo, las políticas de tenencia de animales siguen orientadas a la protección y cuidado de los animales, y minoritariamente a educar y proteger la relación de las personas con los animales y su medio ambiente. Por otro lado, pocas veces tienen asignación de presupuesto y regulación de los estados, pudiendo inferirse que muchas de las normativas existentes no se desarrollan a cabalidad.

\section{Instrumentos legales en Chile}

A nivel nacional existen disposiciones legales que abordan tópicos de TRA de una manera genérica, a partir de las cuales se han generado acciones y actividades para tratar las problemáticas de salud pública que conlleva, los que se traducen a nivel municipal en funciones, ordenanzas e intervenciones focalizadas en campañas sanitarias. Entre los instrumentos se encuentran:

- Código Penal en el Artículo 291 Bis.

- Código Sanitario (Decreto con fuerza de ley №75/1968).

- Reglamento para la prevención de la Rabia en el hombre y animales (Decreto 1/2014).

- Ley sobre protección de animales (Ley $\mathrm{N}^{\mathrm{o}}$ 20.380/2009).

- Reglamento para el control reproductivo de animales de compañía (Decreto 2/2015).

Actualmente, está en trámite un Proyecto de ley de Tenencia Responsable de Animales (Boletín 6499-11), que ha tenido bastantes modificaciones desde sus inicios y en el presente tiene por objeto "promover la tenencia responsable y proteger la salud de mascotas y animales de compañía; proteger la salud pública mediante el control de la población de éstos, y regular la responsabilidad por los daños que sean consecuencia de la acción de dichos animales". Por otro lado, desde el año 2005 surgen diversos dictámenes de la Contraloría para delimitar las atribuciones de las municipalidades respecto al manejo de los perros.

Además instituciones como Carabineros de Chile y Policía de Investigaciones reciben denuncias de maltrato animal, sobre animales sueltos en la vía pública, sobre responsabilidad de dueños de animales y otras problemáticas relacionadas al tema, que van desde cientos a miles de acciones legales al año (Institutio Nacional de Estadísticas, 2011b, 2011a, 2012a, 2012b, 2013a, 2013b, 2014b, 2014a, 2015b, 2015a), sin embargo, algunas son investigadas y otras no prosperan.

\section{Políticas Públicas}

En los países donde existen políticas sobre manejo de población animal, se han establecido programas principalmente a nivel local financiados por el gobierno central, el $73 \%$ de estos países los trabaja mediante la gestión de municipalidades; y, de este porcentaje, el $49 \%$ trabaja en conjunto municipios con ONG y el $23 \%$ restante como entidades solas (Dalla Villa et al., 2010).

Si bien existen distintos enfoques para la formulación de políticas, en función del contexto y su propósito, el modelo comúnmente adoptado dentro del campo de la ciencia política se denomina el ciclo de políticas (Young y Quinn, 2002). El entendimiento de este proceso es relevante para abordar la necesidad de construcción de una política pública para TRA. Explorar en las etapas iniciales del ciclo (planteamiento del problema), puede contribuir a identificar las limitaciones y oportunidades en Chile para aproximarse a una TRA desde un enfoque integral. Con ello se pueden sustentar las bases para sostener el trabajo en una agenda que en la actualidad no ha sido clara en sus lineamientos, o que el paso de la problemática de la agenda pública a la institucional en temas de animales de compañía no se concreta, pese a que los inconvenientes son conocidos o públicos y afectan tanto a las personas como al medio ambiente (Soto, 2013).

Luego de revisar las distintas perspectivas que pueden ser adoptadas para valorar la tenencia de animales, de revisar los lineamientos y recomendaciones de organismos competentes en la materia e identificar los instrumentos que se han desarrollado en otros países y en Chile, el presente estudio 
busca contribuir en la formulación de una política en torno a la TRA que se implemente a nivel local, identificando los ámbitos temáticos que valoran los diferentes actores sociales involucrados en la problemática de la tenencia animal y las acciones de mayor consenso para los tomadores de decisión o decisiones en el contexto nacional. Con eso se proveerá un insumo que impulse su génesis $\mathrm{y}$, por otro lado, realizará un aporte tanto para su creación, formulación, e implementación en el contexto nacional.

\section{Metodología}

El diseño del estudio combinó métodos de Revisión Sistemática de literatura científica y gris para identificar las acciones y estrategias relacionadas al enfrentamiento de la TRA. En un segundo momento, se utilizó una metodología de consulta a actores sociales claves de la esfera nacional, para consensuar los contenidos que emergieron de la Revisión de literatura (Figura 1).

\section{Revisión de Literatura y documenta- ción.}

La revisión consideró las recomendaciones internacionales para asegurar la calidad de la revisión (Levac et al., 2010, Higgins y Green, 2011). Se utilizaron términos claves para efectuar una búsqueda amplia de documentación científica y literatura gris. No se consideró el resultado de evaluaciones, la efectividad $\mathrm{u}$ otras estimaciones de costo beneficio de las estrategias o acciones. Se realizó entre los meses de septiembre y noviembre del 2013.

\section{Términos de búsqueda}

Se utilizaron como términos claves: Tenencia, Responsabilidad, Cuidado, Bienestar, Protección, Propiedad, Prácticas; a los cuales se sumaron los términos Animal de Compañía, Mascota, Perro, Canino, Gato, Felino para delimitar la estrategia a grupos de animales. Se buscaron los términos apropiados para análogos en idioma inglés. La búsqueda se efectuó en textos en idioma español, inglés y portugués. Se incluyeron textos relacionados a los términos de búsqueda y se excluyeron textos sin datos suficientes sobre autor(es), lugar, vigencia dentro de la temporalidad de la búsqueda, textos de animales diferentes a caninos y felinos, de animales con otras utilidades como animales de experimentación, animales de producción, especies distintas a caninos y felinos domésticos, artículos clínicos, artículos repetidos con otro buscador y los que no fueron atingentes a los propósitos de la revisión.

\section{- Documentos no científicos}

Se recurrió a los buscadores Google, Google Scholar, repositorios digitales, institucionales (OIE) y portales de organizaciones. Se revisó cada documento de manera completa, se elaboró una ficha por documento y posteriormente una matriz. Se seleccionaron un total de 13 documentos publicados entre los años 2001 y 2013.

\section{- Documentos científicos}

Se emplearon buscadores multidisciplinarios como Redalyc, Pubmed, BS-LILACS y Knowledge. Los artículos seleccionados fueron revisados en el formato de abstract, se confeccionó una matriz con las fichas de cada documento. Se incluyeron un total de 75 textos científicos publicados desde enero de 2004 a octubre de 2013.

Finalmente, se elaboró un resumen con una síntesis de los contenidos extraídos de la revisión de literatura gris y científica, donde primeramente se incorporaron todas las estrategias extraídas y en seguida fueron analizadas.

\section{Metodología DELPHI}

Con el objetivo de extraer información a partir de informantes claves, se utilizó la metodología DELPHI modificada (Steurer, 2011, Varela-Ruiz et al., 2012, Hsu y Sandford, 2007), dada su utilidad demostrada en la recolección y síntesis del conocimiento, y la opinión de expertos frente a temas complejos para los cuales se requiere llegar a un consenso o formar una opinión general, particularmente cuando la información existente no es exacta, o carece de objetividad como es el caso de los temas de TRA en Chile. Se llevó a cabo en el período de enero a marzo de 2014.

\section{- Muestra de participantes}

La muestra fue por conveniencia. Se identificaron informantes claves con residencia en las 15 regiones de Chile, mayores de 25 años, que tuvieran interés y una trayectoria en el tratamiento de problemáticas de cuidado animal, o participación y/o responsabilidad en implementación de actividades de protección del ambiente y salud de las personas. Se conformó una 
Figura 1: Diseño del estudio

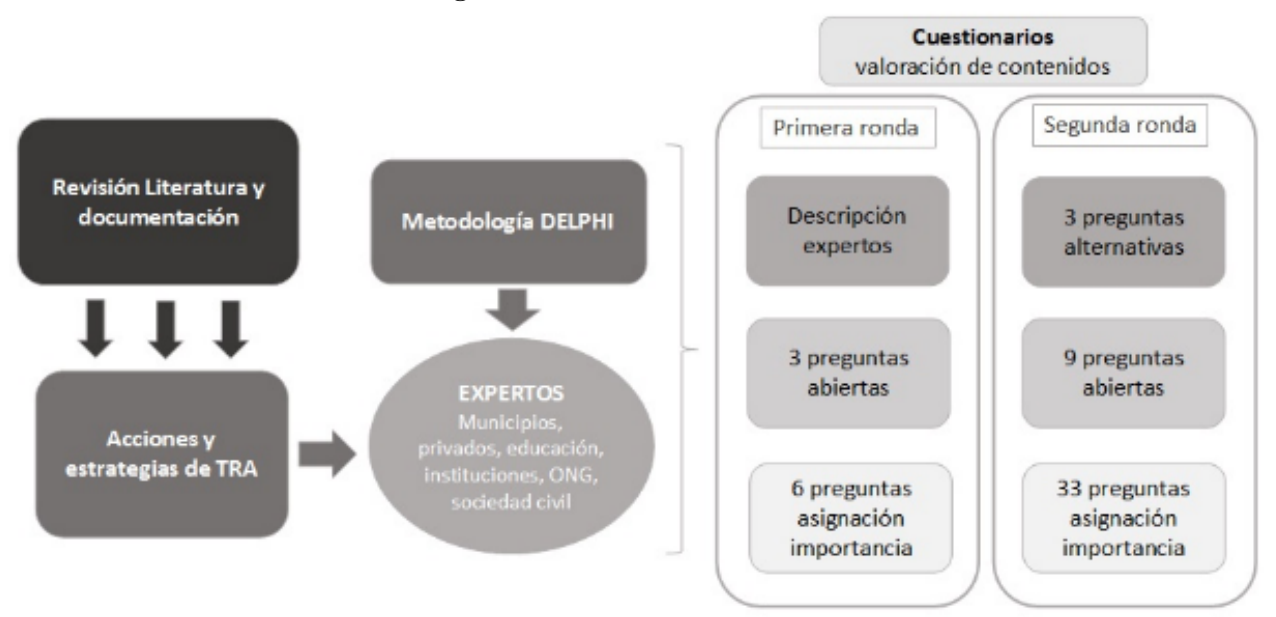

Fuente: Elaboración propia

base de datos con 64 potenciales participantes, se recibieron 22 respuestas con intención de participar en el estudio, a quienes se les entregó el consentimiento informado.

\section{- Cuestionarios}

Se diseñó un primer cuestionario semiestructurado, se utilizó un formato online (Google Docs) e indicaciones para un correcto llenado. Las preguntas se elaboraron con el objeto de recoger la valoración de las grandes acciones de TRA sometidas a evaluación pudiendo cada participante adicionar elementos no presentados. El método se desarrolló en forma anónima, consideró una iteración y realimentación controlada.

Posteriormente, se diseñó un segundo cuestionario semi-estructurado. empleándose el mismo formato online. Se valoraron las estrategias correspondientes a las acciones de TRA, pudiendo adicionar estrategias no presentadas y asignando roles o responsabilidades a distintas entidades relacionadas con la temática. Todos los participantes recibieron un resumen de resultados y un certificado de participación en el estudio.

El estudio fue aprobado por el Comité de Ética de la Facultad de Medicina.

\section{Análisis de la información}

- Análisis revisión literatura

En la revisión de documentos científicos y de literatura gris se efectuó análisis de contenidos. Durante el análisis se identificaron y extrajeron todas las estrategias enunciadas de los documentos, para luego enumerarlas y agruparlas en una sola matriz. Las estrategias o actividades para la TRA fueron evidenciadas por su mención, independiente de la calificación positiva o negativa en cada texto, y de la validez que tenían en el contexto de cada documento.

- Tratamiento de Cuestionarios

Para las preguntas abiertas se efectuó análisis de contenidos, para cada una de ellas se elaboró una matriz con las respuestas entregadas por los participantes, luego fueron revisadas y analizadas una a una hasta obtener un nivel de saturación de los contenidos. En las preguntas orientadas a la identificación de estrategias, se confeccionaron listas enumeradas de las diferentes ideas, cuando existieron se agruparon los argumentos y se analizaron los contenidos. En las preguntas orientadas a la valoración, también se ordenaron las respuestas en una matriz y fueron categorizadas en grupos de argumentos a favor y en contra. En las preguntas con escala, se evaluó el grado de importancia y se calcularon frecuencias de respuestas. En las preguntas de alternativas, se calcularon frecuencias de respuestas.

\section{Resultados}

\section{Resultados Revisión Literatura}

A partir del análisis de contenidos de la literatura se extrajeron 6 categorías que conformaron los tópicos preliminares de "acciones" que componen un Programa de TRA. Dichas acciones fueron definidas sobre la base de los propósitos enunciados en la literatura revisada para identificar posterior- 
mente 50 estrategias operativas agrupadas en cada dominio de acción (Tabla 1).

Existe un buen sustento documental referente a las temáticas abordadas por la tenencia responsable de animales a nivel internacional. Los 6 tópicos fueron definidos en relación a su utilidad para trabajar la tenencia responsable de manera integral, y donde si bien las estrategias específicas de cada uno están orientadas hacia un objetivo particular, ellas pueden relacionarse con las estrategias de las otras acciones.

La acción 1, que es la de mayor conocimiento general y la más documentada, debido a que varias de sus estrategias son habituales de la práctica del ejercicio veterinario, en este caso se amplía, de tener inicialmente fines casi exclusivamente sanitarios, a abordar temas contemporáneos como lo son el bienestar animal y la inclusión de animales en situaciones de emergencias y desastres; asunto escasamente abordado en los países.

La acción 2 de control de población, al igual que la anterior está centrada esencialmente en los animales, en ella aparecen estrategias que han ido evolucionando a través del tiempo, por ejemplo herramientas como el sacrificio de perros fue una práctica habitual en Chile en la década de los 50', y hoy se utiliza el término "eutanasia" de los animales; que implica no sólo el deceso del animal, sino que con el menor sufrimiento posible. Además, con los avances y nuevas tecnologías hoy se habla de sistemas de identificación únicos, mediante dispositivos electrónicos instalados en los animales para el manejo y control de poblaciones animales, que son priorizadas en programas internacionales, complementarias a otras estrategias e implementadas cuando ya existe un respaldo legal para su uso (Dalla Villa et al., 2010).

La acción 3 está centrada en las personas, abarca soluciones que tienen que ver con la modificación de actitudes de las personas hacia los animales, incorporando herramientas de educación formal y no formal, otras de carácter cultural; incluyendo áreas profesionales diferentes a veterinaria, además de ésta; y a otros grupos de la sociedad que permitan producir los cambios en la convivencia con animales de compañía. Si bien, es un tópico poco abordado en los países, es necesario no sólo para crear conciencia de los cuidados requeridos por los animales, sino que porque la relación con ellos tiene un riesgo intrínseco en la transmisión de enfermedades por ejemplo. Por otro lado, se hace mención a los medios de comunicación, que son bastante influyentes en algunos grupos de la sociedad, produciendo cambios de comportamiento y de actitudes.
La acción 4 incorpora no sólo la existencia de una orgánica para la TRA, sino que plantea herramientas que permitan conocer de mejor manera los escenarios previos a las intervenciones y los fenómenos de las poblaciones animales, que posibilitan en los tomadores de decisiones la planificación y coordinación de las actividades; y otras para desarrollar una gestión eficiente de un programa de esta índole.

La acción 5 tiene estrategias dirigidas tanto a las personas como a los animales, pretende proteger el entorno y mitigar los efectos de las conductas inapropiadas de la tenencia animal, son las menos documentadas, pero no menos importantes para trabajar la TRA.

La acción 6 corresponde al sustento legal para poder resguardar la implementación de las acciones de TRA, en un Programa que se pueda mantener en el tiempo y normar las conductas de las personas en la tenencia de animales.

\section{Resultados consulta a expertos}

Se obtuvo un $23 \%$ de la muestra convocada. Las causales de no participación fueron indeterminadas en la mayoría de los casos, puesto que 42 de los convocados no dieron respuesta a la convocatoria. Quienes enviaron el consentimiento y participaron voluntariamente, lo hicieron tanto en la primera como en la segunda ronda, logrando una tasa de retorno de los cuestionarios de $100 \%$.

La muestra quedó conformada por participantes entre 26 y 50 años de edad (37 años promedio), la mayoría profesionales mujeres, residentes en 12 comunas urbanas y rurales, pertenecientes a 8 regiones de Chile (Antofagasta, Coquimbo, Metropolitana, Bío-Bío, Araucanía, Los Lagos, Aysén del General Carlos Ibáñez del Campo, Magallanes y de la Antártica Chilena). Catorce informantes son profesionales universitarios, en su mayoría Médicos Veterinarios (as) (n=11) y uno técnico. Entre los cargos que ocupan siete son referidos a funciones de jefaturas o cargos de dirección pública en el ámbito de la salud pública, tres a dirección o presidencia de fundaciones sin fin de lucro u ONG. Participaron tres representantes del mundo académico público y privado, un profesional independiente de clínica privada, un asesor legal de proyectos y políticas ligados a la temática animal, y dos funcionarios públicos que realizan asistencia y apoyo profesional, uno en entidad relacionada con las ciencias y otro en agricultura. Respecto a la trayectoria, describen experiencia promedio de 7 años en temas relacionados a TRA.

Sólo el $50 \%$ de los contenidos extraídos desde la 
Tabla 1: Matriz de acciones y estrategias de TRA extraídas de literatura

\begin{tabular}{|c|c|c|}
\hline Acciones & Definición & Estrategias \\
\hline \multirow{7}{*}{$\begin{array}{l}\text { Acción } 1 . \\
\text { Promover la } \\
\text { salud, } \\
\text { cuidado y } \\
\text { bienestar de } \\
\text { las personas y } \\
\text { de los } \\
\text { animales de } \\
\text { compañía. } \\
\end{array}$} & \multirow{7}{*}{$\begin{array}{l}\text { Corresponde a las acciones preventivas y } \\
\text { curativas de fomento de la salud de los } \\
\text { animales de compañía y orientadas a } \\
\text { disminuir el contagio de enfermedades } \\
\text { desde los animales hacia las personas. } \\
\text { Además de la preocupación sobre la } \\
\text { calidad de vida de las mascotas y sus } \\
\text { necesidades generales o básicas. Atención } \\
\text { veterinaria preventiva y curativa. }\end{array}$} & $\begin{array}{l}\text { Asesoramiento veterinario a propietarios para cuidados genera- } \\
\text { les de las mascotas. }\end{array}$ \\
\hline & & $\begin{array}{l}\text { Requisitos para lugares donde se realice confinamiento de mas- } \\
\text { cotas (hoteles, clínicas, refugios, tiendas, criaderos). }\end{array}$ \\
\hline & & Captura y albergue de mascotas. \\
\hline & & Aplicación de eutanasia sobre la base a distintos criterios. \\
\hline & & Atención de especialistas en conducta animal. \\
\hline & & Supervisión y sujeción de animales en espacios públicos. \\
\hline & & Asistencia veterinaria en emergencias y desastres. \\
\hline \multirow{10}{*}{$\begin{array}{l}\text { Acción } 2 . \\
\text { Control de la } \\
\text { población de } \\
\text { animales de } \\
\text { compañía. }\end{array}$} & \multirow{10}{*}{$\begin{array}{l}\text { Corresponde a las acciones que permiten } \\
\text { manejar y controlar la población de } \\
\text { animales de compañía, ya sea para regular } \\
\text { la cantidad de animales, identificar la } \\
\text { procedencia de ellos, conocer quienes son } \\
\text { los responsables o supervisores de ellos y } \\
\text { otras características. }\end{array}$} & $\begin{array}{l}\text { Control de la reproducción de animales de compañía, esteriliza- } \\
\text { ción. }\end{array}$ \\
\hline & & Sistema de identificación de mascotas. \\
\hline & & Sistema de registro de mascotas. \\
\hline & & Eliminación, eutanasia de mascotas. \\
\hline & & Adopción. \\
\hline & & Resguardo y control de animales en viviendas. \\
\hline & & Permiso para tenencia de animales. \\
\hline & & Albergues/refugios. \\
\hline & & Entrega voluntaria a centros de recepción de mascotas. \\
\hline & & Captura de animales en las calles. \\
\hline \multirow{8}{*}{$\begin{array}{l}\text { Acción } 3 . \\
\text { Realizar } \\
\text { educación } \\
\text { sanitaria y } \\
\text { ambiental. }\end{array}$} & \multirow{8}{*}{$\begin{array}{l}\text { Corresponde a las acciones que permitan } \\
\text { difundir información y educar a la } \\
\text { población sobre las prácticas adecuadas } \\
\text { para la tenencia de animales en los } \\
\text { ámbitos sanitario y ambiental. }\end{array}$} & Guías, manuales o instructivos de TRA. \\
\hline & & Inserción de contenidos de TRA en mallas curriculares. \\
\hline & & Campañas de promoción de TRA en medios de comunicación. \\
\hline & & Capacitación sobre TRA a profesores. \\
\hline & & Capacitación sobre TRA a profesionales de salud. \\
\hline & & Capacitación sobre TRA a diversos actores sociales. \\
\hline & & Jornadas, charlas y ferias informativas de TRA. \\
\hline & & Espacios culturales y recreativos para fomento de la TRA. \\
\hline \multirow{11}{*}{$\begin{array}{l}\text { Acción } 4 . \\
\text { Organizar las } \\
\text { funciones, } \\
\text { servicios y } \\
\text { responsabili- } \\
\text { dades de las } \\
\text { instituciones. }\end{array}$} & \multirow{11}{*}{$\begin{array}{l}\text { Corresponde a la gestión de acciones que } \\
\text { se realizan por parte de instituciones y } \\
\text { organizaciones preocupadas de la salud de } \\
\text { las personas y animales, que permiten } \\
\text { orientar de mejor manera la elección de } \\
\text { acciones de tenencia de animales en los } \\
\text { diferentes contextos. }\end{array}$} & Conocer directrices de programas de TRA. \\
\hline & & Caracterización demográfica de población animal. \\
\hline & & Caracterización propietarios de mascotas. \\
\hline & & Diagnósticos locales de salud relacionados con la TRA. \\
\hline & & Creación de Unidad coordinadora de TRA. \\
\hline & & Capacitación continua de unidad de TRA. \\
\hline & & Sistema centralizado denuncias maltrato animal. \\
\hline & & Sistemas de información centralizados sobre TRA. \\
\hline & & $\begin{array}{l}\text { Marco que delimite funciones, atribuciones y responsabilidades } \\
\text { institucionales para la TRA. }\end{array}$ \\
\hline & & Participación de agentes comunitarios en actividades de TRA. \\
\hline & & Formulación de indicadores de evaluación de planes de TRA. \\
\hline \multirow{7}{*}{$\begin{array}{l}\text { Acción } 5 . \\
\text { Cuidar y } \\
\text { promover la } \\
\text { calidad del } \\
\text { ambiente } \\
\text { urbano, rural } \\
\text { y silvestre. }\end{array}$} & \multirow{7}{*}{$\begin{array}{l}\text { Corresponde a las acciones que permiten } \\
\text { disminuir el impacto en el ambiente de las } \\
\text { acciones humanas relacionadas con la } \\
\text { tenencia de animales de compañía. Y para } \\
\text { controlar la tenencia de animales } \\
\text { silvestres como mascotas. }\end{array}$} & $\begin{array}{l}\text { Fiscalización comercio y tráfico animales silvestres y exóticos } \\
\text { para uso como mascotas. }\end{array}$ \\
\hline & & Control de caninos y felinos asilvestrados. \\
\hline & & Control de ingreso de mascotas a parques y reservas nacionales. \\
\hline & & Vigilancia de animales silvestres. \\
\hline & & Control de desechos animales y basura de espacios. \\
\hline & & Manejo y disposición de residuos en espacios públicos y privados. \\
\hline & & Protección y resguardo de sitios desocupados. \\
\hline \multirow{6}{*}{$\begin{array}{l}\text { Acción } 6 . \\
\text { Legislar para } \\
\text { promover la } \\
\text { Tenencia } \\
\text { Responsable } \\
\text { de Animales. }\end{array}$} & \multirow{6}{*}{$\begin{array}{l}\text { Corresponde a la creación de decretos, } \\
\text { leyes o cualquier instrumento normativo } \\
\text { que permita promocionar y regular en la } \\
\text { población la Tenencia Responsable de } \\
\text { Animales. }\end{array}$} & Marco legal sobre TRA (Leyes, reglamentos, ordenanzas). \\
\hline & & Establecer deberes y obligaciones a propietarios de mascotas. \\
\hline & & Restricciones para la tenencia de animales. \\
\hline & & Sanciones a la mala tenencia de animales. \\
\hline & & Multisectorialidad en responsabilidades de la TRA. \\
\hline & & Regulación y fiscalización de actividades de TRA. \\
\hline
\end{tabular}

Fuente: Elaboración propia a partir de la revisión de la documentación científica y no científica 
literatura fueron mencionados por los participantes en formato de pregunta abierta. Se destaca un conocimiento más amplio sobre estrategias de la acción 6 (legislación) y uno menor en relación a estrategias de la acción 3 (educación). Relevan la necesidad de incluir en un Programa temas de salud y bienestar animal, control sanitario de las mascotas (principalmente de zoonosis), control de población de los animales (con énfasis en la esterilización), el registro e identificación de los animales, la educación sanitaria a distintos grupos de la población, asimismo mencionan la promulgación de leyes y la creación de una unidad especializada en el tema.

Los participantes destacan el escaso desarrollo de actividades para la promoción de la TRA, y la respuesta reactiva de acciones ante noticias contingentes que destacan los medios de comunicación. Los participantes mencionan que, a nivel local, sólo algunos municipios efectúan ciertas actividades particulares; esterilizaciones e identificación de animales, pero de manera insuficiente. Se acentúa la falta de educación a la población y la necesidad de crear una política pública.

En la valoración efectuada por los participantes a cada gran acción se evidencia que existe consenso en la incorporación de ellas para la implementación de un Programa de TRA (Figura $2^{1}$ ). Todas alcanzan una alta puntuación, no obstante, se distingue que principalmente las acciones 2 y 6 alcanzan el mayor consenso, seguidas por la acción 3.

\section{Acción 1}

Las estrategias que alcanzaron el máximo consenso entre los participantes fueron la necesidad de establecer un plan de asistencia veterinaria en situaciones de emergencias y desastres, establecer requisitos de funcionamiento en lugares donde existe acopio de animales (refugios, hoteles u otros), y proporcionar a los propietarios de mascotas asesoramiento sobre los cuidados generales de sus animales (Figura 3).

Por el contrario, las estrategias de menor consenso y alta controversia en los argumentos entregados, fueron la práctica de eutanasia, principalmente en animales que presentan zoonosis y animales diagnosticados como agresivos; la captura y confinamiento de animales y el acceso a especialistas en conducta animal.

El establecimiento de un plan de asistencia para los animales en emergencias y desastres fue considerado muy relevante, debido a que en esos escenarios "se producen muchos abandonos" con consecuen-

\footnotetext{
${ }^{1}$ Las acciones se encuentran abreviadas en la figura
}

cias desfavorables para la salud y bienestar de los animales, personas y medio ambiente; además se destaca que es un "deber ético de la sociedad". Los participantes plantean la necesidad de que "deben existir equipos de rescate y asistencia, capacitados y remunerados con el desarrollo de un trabajo articulado con fundaciones, universidades y profesionales del ámbito veterinario" para dar respuesta en estos contextos.

Asimismo se justifica la existencia de requisitos con estándares de condiciones óptimas para el mantenimiento de los animales en el funcionamiento de tiendas de mascotas, albergues, hoteles, criaderos y otros lugares donde exista confinamiento de los animales, ámbitos en los que se denota la necesidad de "regular y fiscalizar estos estándares para mantener el bienestar de los animales y evitar problemas sanitarios". Se sugiere también determinar "la esterilización de todos los animales que se adquieran de albergues y criaderos", así como "fijar límites en la cantidad de animales en cada uno de estos lugares", cómo ámbitos a regular.

La entrega de asesoría a la población en manejos y cuidados básicos de los animales de compañía es planteada como acción "complementaria en la asistencia preventiva", destacando "la falta de conocimiento del comportamiento de ellos" como una de las principales causas de abandono. En este sentido, es planteado que el acceso a especialistas en conducta animal ante la presencia de conductas indeseadas, favorecería la prevención del abandono, mordeduras y accidentes; sin embargo, "debiese ser un costo asumido por los propietarios y sólo asumir un rol de ayuda financiera municipal en casos demostrados de carencias de recursos económicos".

Con respecto a los desacuerdos en determinadas estrategias, particularmente en la implementación de captura y confinamiento de animales en albergues, se evalúa como una medida que "podría conducir a un mayor nivel de hacinamiento, con incremento de problemas sanitarios en los animales e incentivar la eutanasia" debido a la imposibilidad de reubicar a los animales capturados. Otros efectos colaterales y negativos que se argumentan se refieren a lo "oneroso" de la medida, a la "imposibilidad de fiscalizar", además de "fomentar el abandono" de animales por parte de los propietarios y con ello "favorecer formas de maltrato animal e irresponsabilidad en los propietarios". No obstante, hubo participantes que creen que los albergues podrían ser de utilidad en algunas localidades como medida de "mitigación de riesgos para la comunidad", por ejemplo, con el "retiro de animales agresivos y hembras en celo de la vía pública", y que ellos podrían "ser asistidos, vacunados, identificados, esterilizados y posteriormente decidir su destino". 
Figura 2: Ámbitos de acción de TRA y percepción de nivel de importancia. El $\mathrm{N}^{\circ}$ de asignaciones son participantes

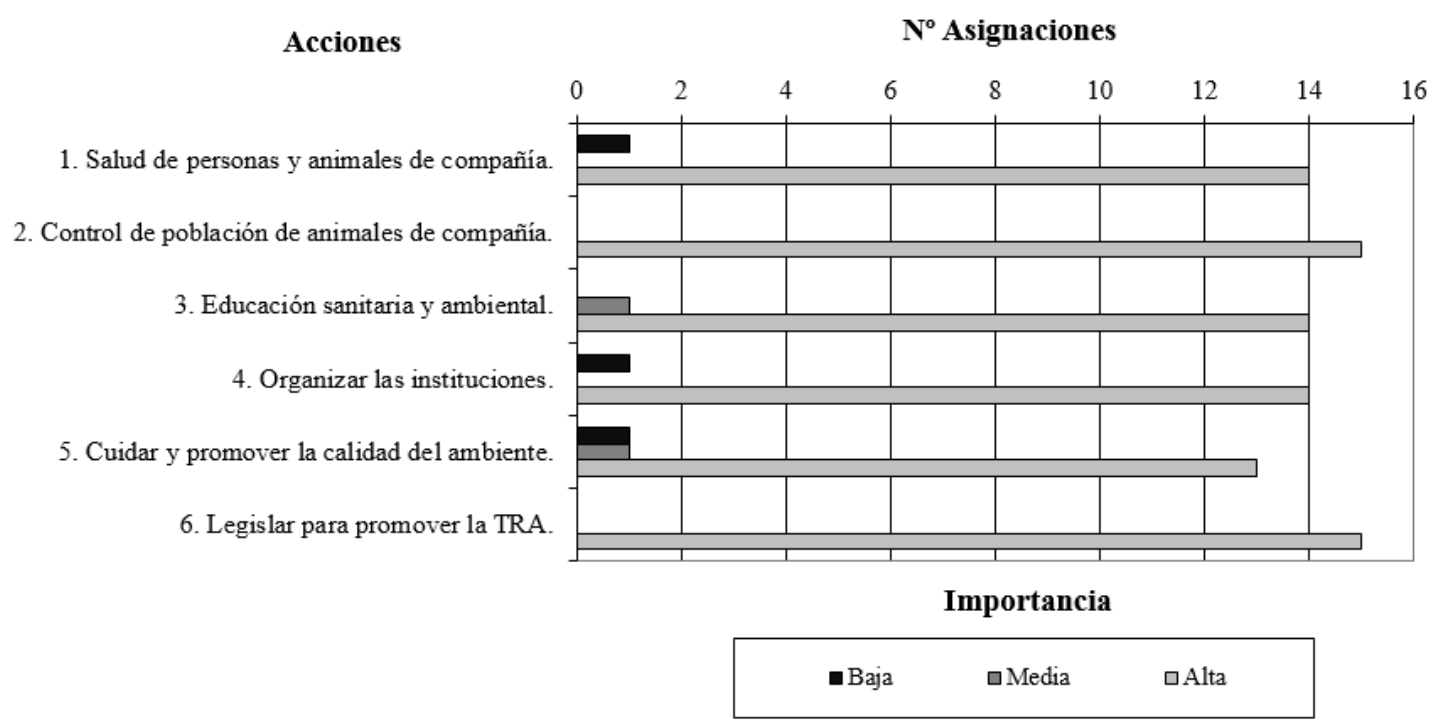

Fuente: Elaboración propia

Figura 3: Estrategias de acción 1 para la TRA y su percepción por nivel de importancia. El № de Asignaciones son participantes

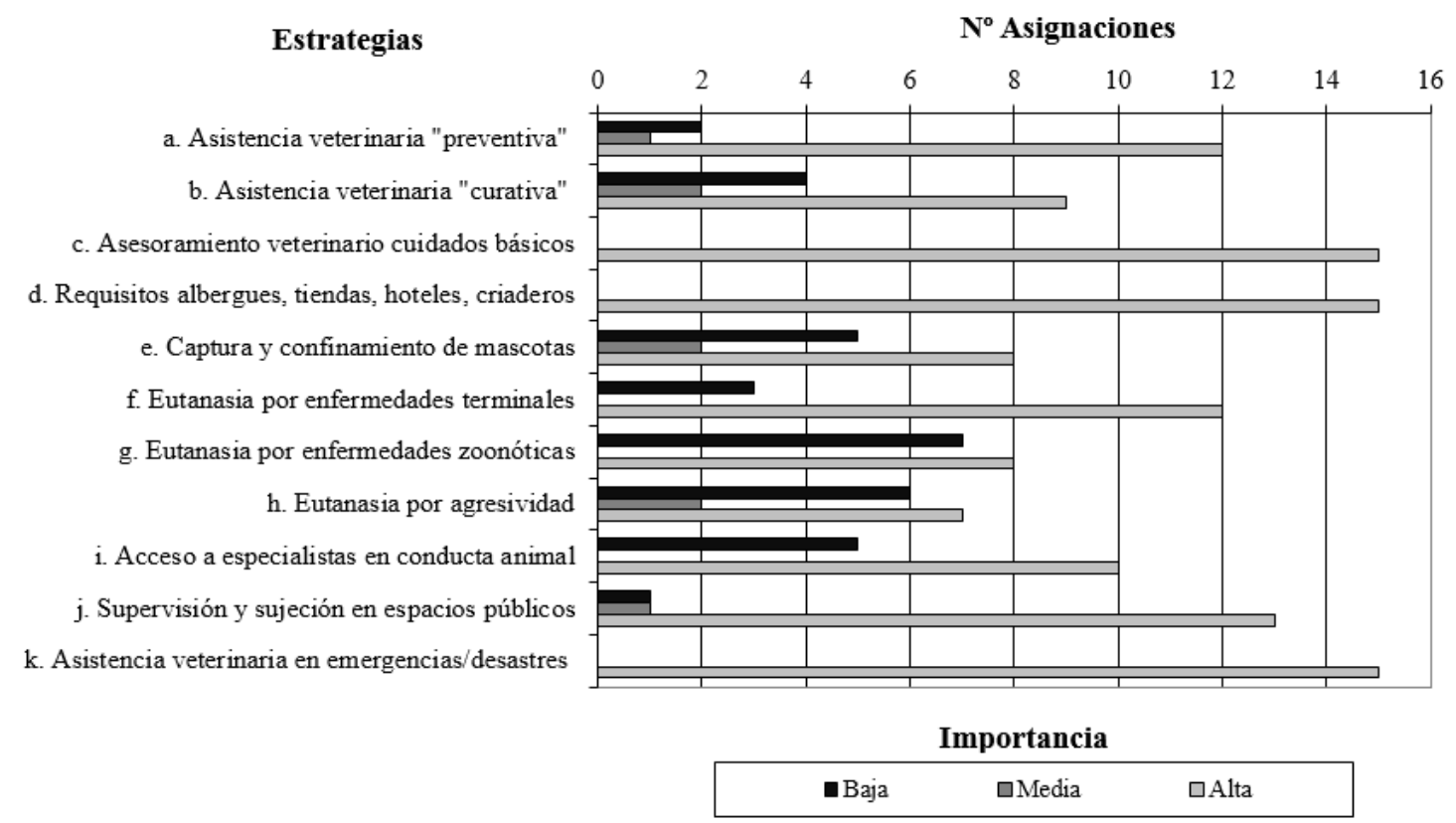

Fuente: Elaboración propia 
Frente a la práctica de eutanasia como procedimiento no existe acuerdo sobre su incorporación a un programa de TRA, principalmente en los casos de zoonosis y agresividad. Respecto a la eutanasia los expertos mencionan que se debe tener cuidado con el "uso arbitrario, indiscriminado y descontrolado de esta medida por parte de los municipios" y que "no debe ser usada como control de población". Pese al bajo consenso general de esta estrategia, existen argumentos que se indican a favor de esta medida, y que, en el caso de ser aplicada, "debiese orientarse a animales con enfermedades terminales o con un gran deterioro de su calidad de vida". El criterio a aplicar por parte del Médico Veterinario es relevado, así como también el uso de técnicas indoloras. Para el caso de las zoonosis se señala que "es importante la educación para profesionales del área de la salud y veterinarios en esta materia como herramientas de prevención". En el caso de practicar eutanasia en situación de zoonosis ésta "sería de utilidad sólo para los casos de rabia", ya que para otras zoonosis existen tratamientos que pueden revertir el estado de esos animales. Argumentos similares emergen para los animales calificados como agresivos que "pueden ser tratados y rehabilitados".

\section{Acción 2}

Entre las estrategias de mayor consenso destaca la necesidad de incorporar la formación de centros de esterilización de animales y que se practique la identificación y registro de animales (Figura 4). Argumentan la necesidad de centros de esterilización de animales, "tanto de machos y hembras para prevenir la aparición de nuevas camadas", algunos le dan un carácter "gratuito y universal", otros de "esterilización obligatoria a caninos y felinos" y otros "a precios rebajados". Además destacan que debe efectuarse la esterilización de "todos los animales que vayan a ser adquiridos por un propietario".

Por otro lado, la existencia de un sistema de registro e identificación de animales se considera prioritaria en el control del abandono animal. Dado que constituye una herramienta fundamental para el cumplimiento de ordenanzas y aplicación de sanciones, se alude a la necesidad de crear un sistema de subvención para solventar su implementación junto a la implementación de esterilización de animales en sectores socialmente vulnerables. Los expertos recalcan el hecho de que las actividades descritas para el control de población deben ser complementadas indiscutiblemente con educación a la población, además de tener el respaldo legal para la fiscalización y con ello dotar de personal capacitado para su implementación.
Aspectos menos valorados sobre su utilidad como control de la población animal, fueron la creación de centros para captura, recepción y entrega voluntaria de animales, destacando como argumento la "ineficacia e ineficiencia" de la medida debido a que el "origen de la falta de control de las poblaciones radica en los animales que tienen dueño y que salen a la vía pública sin supervisión". También fue evaluada de baja utilidad la eutanasia como medida de control de la población animal.

\section{Acción 3}

Los participantes destacan dos estrategias fundamentales para el fomento de la TRA (Figura 4). La primera corresponde a la inclusión de contenidos en la formación curricular escolar (primaria y secundaria), pues ésta constituiría una vía para "proveer educación permanente en etapas tempranas a niños y niñas", además de permitir tratar las temáticas de tenencia responsable transversalmente en los planes de estudio y lograr además una mayor cobertura. Esta indicación debe ir acompañada de acuerdo a los participantes de la capacitación de los profesores, que son los que están en contacto estrecho diariamente con niños, niñas y jóvenes para "producir a futuro un cambio cultural y comportamental". Una segunda acción muy valorada es la realización de campañas de difusión masivas en medios de comunicación, existiendo consenso en que tenga un carácter nacional para lograr un mayor impacto en la población.

Según los participantes, los principales temas que debiesen ser incluidos en educación son los siguientes (en orden de importancia):

- Zoonosis.

- Control de reproducción de las mascotas.

- Necesidades, manejos y cuidados generales de las mascotas.

- Legislación chilena relacionada con TRA.

- Bienestar animal.

\section{Acción 4}

Las estrategias con mayor consenso fueron la necesidad de que exista un marco regulador que norme el funcionamiento de un Programa de TRA y defina sus alcances, así como la necesidad de creación de una unidad especializada de TRA a nivel municipal con acceso a capacitación continua. 
Figura 4: Número de preferencias para estrategias de la acción 2

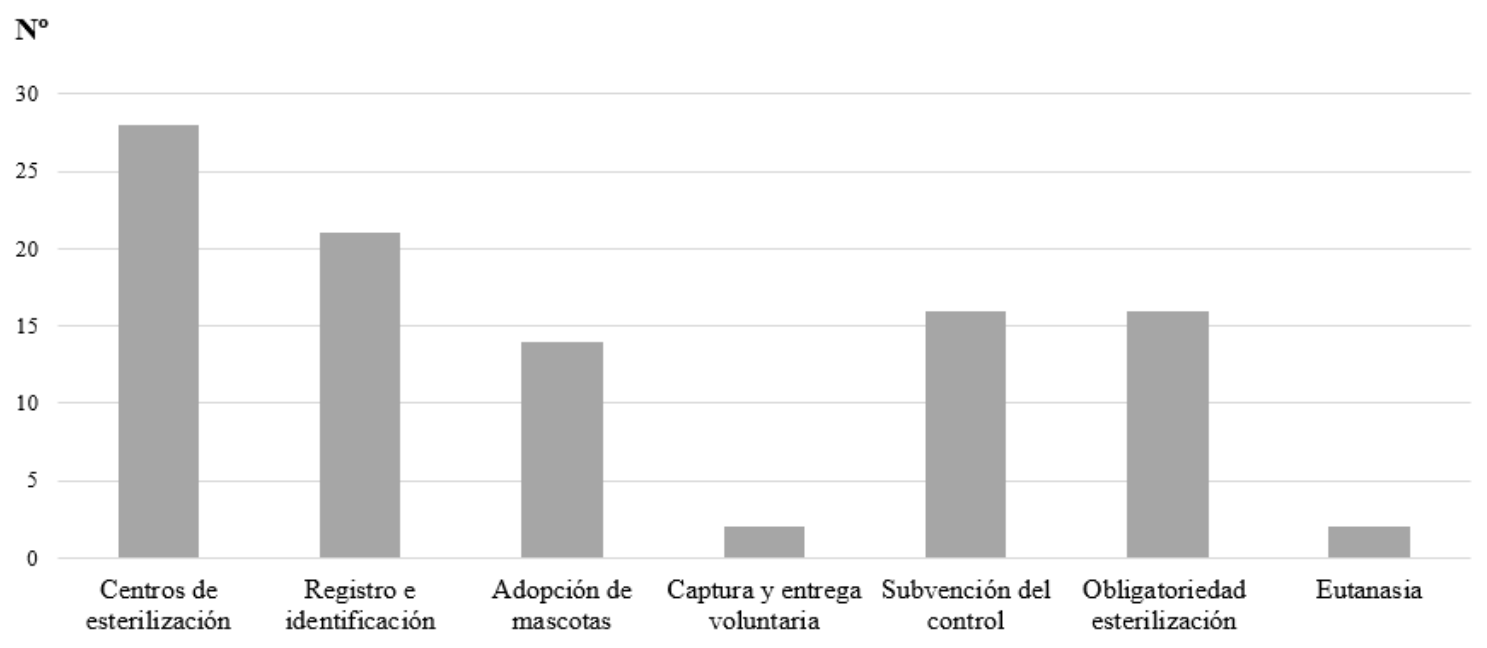

\section{Estrategias}

Fuente: Elaboración propia

Figura 5: Número de preferencias para estrategias de la acción 3

$\mathbf{N}^{\circ}$

35

30

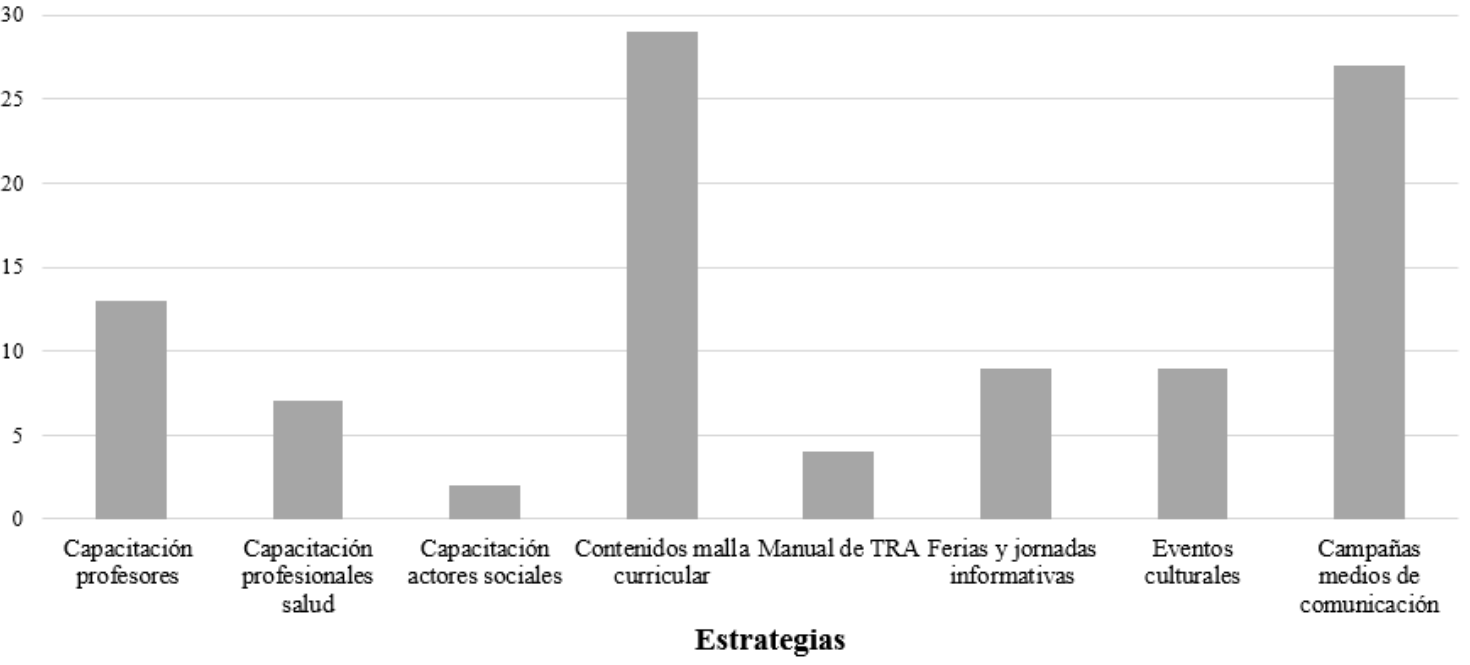

Fuente: Elaboración propia 
Respecto al marco regulador, los expertos mencionan que su inexistencia actual es "uno de los principales obstáculos para la implementación de un programa que ejecute acciones concretas y fiscalice su cumplimiento". Por otro lado, también advierten que la implementación de un programa de TRA requiere de una unidad con profesionales especialistas, destacando la participación de Médicos Veterinarios y educadores liderando las estrategias. Asimismo, se destaca como muy necesario definir claramente los roles de cada institución y los objetivos de los programas para el fomento de la TRA.

\section{Acción 5}

Las respuestas predominantes en esta acción son tres principalmente, una de ellas corresponde a la necesidad de inclusión de acciones para el control de la población de caninos y felinos asilvestrados, destacando entre las actividades nombradas el retiro de caninos y felinos de vida libre o abandonados en lugares silvestres, enfatizando el uso de "métodos éticos" para estos fines. La fiscalización del ingreso de mascotas a lugares protegidos como reservas y parques nacionales, exceptuando el caso para perros de ayuda (lazarillos). Y por último, existió acuerdo para incluir la fiscalización del manejo y disposición adecuada de basura domiciliaria. En general hubo escasa argumentación por parte de los participantes frente a todas las estrategias presentadas de esta acción.

\section{Acción 6}

Se señalan con claridad diversas temáticas que se deben abordar en el ámbito legal para el fomento de la TRA en Chile, abogando al trabajo intersectorial (ambiente, salud y educación):

- Definición de deberes o responsabilidades de los tenedores de animales de compañía.

- Inclusión de temáticas de TRA en la educación formal.

- Definición de roles institucionales en la TRA.

- Garantía de la asignación de recursos para la implementación de políticas de TRA.

- Aseguramiento del desarrollo de acciones preventivas dentro del Programa.

\section{Responsabilidades}

Se reconocen diferentes roles que deben asignarse a ciertas instituciones para la implementación del programa, sin embargo, no existe una tendencia clara en las respuestas acerca de la institución responsable de éste, se mencionó tanto al Ministerio del Interior, como al Ministerio de Salud y al Ministerio del Medio Ambiente. Sin embargo, para la implementación del Programa, fue notoria la responsabilidad que se le atribuye a las municipalidades.

La colaboración mas relevada por los expertos es la de las organizaciones comunitarias, para la difusión del Programa, entre ellas las juntas de vecinos, ONGs o fundaciones.

\section{Fortalezas y limitaciones del estudio}

Una fortaleza es que la revisión de literatura realizada aportó un fuerte sustento documental internacional en torno a la TRA, y los expertos reconocieron varias de las estrategias recopiladas en ella. Esta revisión fue de gran utilidad para profundizar en detalle en las acciones globales y estrategias, diseñar y nutrir los cuestionarios, lo cual contribuyó y complementó enormemente a la metodología de consulta a expertos (Tetzlaff et al., 2012).

Otra fortaleza es el medio de comunicación utilizado para las consultas, el cual permitió incluir a participantes de diversas regiones y localidades del país, lo cual es valorado en este tipo de metodología (Keeney et al., 2001). La elección de un método presencial en este caso habría dificultado posiblemente la participación de ciertos actores, reduciendo el espectro de opiniones que se recogieron. Es importante destacar el gran interés expresado por los participantes por contribuir con su opinión en el estudio, lo cual se tradujo en respuestas oportunas y completas en los cuestionarios. Los antecedentes mencionados son elementos que aumentan la validez interna de los resultados obtenidos en este estudio (Tetzlaff et al., 2012).

Dentro de las limitaciones metodológicas es que el estudio no puede asegurar la representación de todas las visiones en torno a la TRA en Chile, ya que se desconocen los motivos de los sujetos que no participaron, lo cual imposibilita caracterizar el perfil de rechazo, no obstante, aparentemente éstos no diferirían en ámbitos profesionales de los que sí participaron. Tampoco es factible en esta metodología asegurar la imparcialidad de los participantes frente a la TRA (Keeney et al., 2001). La mayoría de los profesionales que participaron fueron Médicos Veterinarios lo cual restringió las posibilidades de indagar en estrategias en algunos ámbitos, particularmente en educación o en el dominio de la legislación, pero actualmente son el ámbito profesional que trabaja en la temática de TRA. 


\section{Conclusiones}

Se identificaron seis dominios de acción relevados en la literatura revisada que pueden ser parte de un programa de fomento de la TRA, éstos abarcan un conjunto de estrategias en el ámbito de la promoción del cuidado y bienestar de personas y animales, el control de la población animal, la educación sanitaria y ambiental, la legislación en materia de tenencia animal, la organización de responsabilidades institucionales y la promoción del cuidado ambiental. Dichos dominios alcanzan un alto nivel de importancia para ser incorporados en un programa de TRA en Chile, siendo componentes prioritarios a incluir los que apuntan a acciones para el control de población animal, alcanzar un marco normativo para garantizar las estrategias destinadas al fomento de la TRA, y la implementación de un componente educativo. La valoración que entregaron los participantes del estudio, es en suma concordante con las acciones que actualmente se efectúan y priorizan en programas internacionales y entregados en las recomendaciones de la Organización Mundial de Sanidad Animal.

Podríamos decir a través de este estudio que las estrategias sanitarias y de control de población son las más conocidas e implementadas, y las que tienen relación con el cuidado del ambiente son más desconocidas y por lo tanto, menos aplicadas dentro de programas de TRA. Existen actualmente acciones efectuadas a nivel país, principalmente esterilizaciones a nivel local, pero es muy relevante el énfasis que existe de incorporar la educación transversal en los programas de TRA, así como la incorporación de educación formal, destacándose esto como un tema deficitario en el país y necesario ir transformando un cambio cultural paulatino en las personas hacia la TRA. Existen estrategias que generan controversias, principalmente la eutanasia y la conformación de refugios de animales, destacándose los argumentos sobre cuestionamientos éticos y de eficiencia en la aplicación de ellas, para el establecimiento de una tenencia responsable de animales.

Además es posible concluir que falta concretar una política pública nacional sobre TRA, que permita dirigir, alinear y complementar las acciones locales que se desarrollan actualmente. Esta iniciativa lograría poner a Chile a la par con otros países que aparecen como referencia en la aplicación de diversas estrategias de TRA.

\section{Referencias}

(2012). La Institucionalización del Bienestar Animal, un Requisito para su Desarrollo Normativo,
Científico y Productivo. En Gonzalez, G., Stuardo, L., Benavides, D., y Villalobos, P., editores, Actas del Seminario, p. 344, Santiago de Chile.

Broom, D. M. (2011). Animal welfare: concepts, study methods and indicators. Revista Colombiana de Ciencias Pecuarias, 24(3):306-321.

Dalla Villa, P., Kahn, S., Stuardo, L., Iannetti, L., Di Nardo, A., y Serpell, J. A. (2010). Freeroaming dog control among OIE-member countries. Preventive veterinary medicine, 97(1):5863.

Fuentes Cintra, M., Pérez García, L., Suárez Hernández, Y., Soca Pérez, M., y Martinez Martinez, A. (2006). La zoonosis como ciencia y su impacto social. REDVET. Revista Electrónica de Veterinaria, 7(9).

Gómez, L. F., Atehortua, C. G., y Padilla, S. C. O. (2007). La influencia de las mascotas en la vida humana. Revista Colombiana de Ciencias Pecuarias, 20(3):377-386.

Higgins, J. P. T. y Green, S. (2011). Manual Cochrane de revisiones sistemáticas de intervenciones. The Cochrane Collaboration.

Hsu, C.-C. y Sandford, B. A. (2007). The Delphi technique: making sense of consensus. Practical assessment, research $\&$ evaluation, 12(10):1-8.

Institutio Nacional de Estadísticas (2011a). Informe Anual 2010 Carabineros de Chile. INE, Santiago de Chile.

Institutio Nacional de Estadísticas (2011b). Informe Anual 2010 Policía de Investigaciones de Chile. INE, Santiago de Chile.

Institutio Nacional de Estadísticas (2012a). Informe Anual 2011 Carabineros de Chile. INE, Santiago de Chile.

Institutio Nacional de Estadísticas (2012b). Informe Anual 2011 Policía de Investigaciones de Chile. INE, Santiago de Chile.

Institutio Nacional de Estadísticas (2013a). Informe Anual 2012 Carabineros de Chile. INE, Santiago de Chile.

Institutio Nacional de Estadísticas (2013b). Informe Anual 2012 Policía de Investigaciones de Chile. INE, Santiago de Chile.

Institutio Nacional de Estadísticas (2014a). Informe Anual 2013 Carabineros de Chile. INE, Santiago de Chile.

Institutio Nacional de Estadísticas (2014b). Informe Anual 2013 Policía de Investigaciones de Chile. INE, Santiago de Chile. 
Institutio Nacional de Estadísticas (2015a). Informe Anual 2014 Carabineros de Chile. INE, Santiago de Chile.

Institutio Nacional de Estadísticas (2015b). Informe Anual 2014 Policía de Investigaciones de Chile. INE, Santiago de Chile.

Keeney, S., Hasson, F., y McKenna, H. P. (2001). A critical review of the Delphi technique as a research methodology for nursing. International journal of nursing studies, 38(2):195-200.

Levac, D., Colquhoun, H., y O'Brien, K. K. (2010). Scoping studies: advancing the methodology. Implementation Science, 5(1):69.

Ley 13.879 (2008). Ley que establece prácticas de Protección Animal.

Ley 14.346 (1954). Ley de Malos Tratos y Actos de Crueldad a los Animales.

Ley 18.471 (2009). Ley de Tenencia responsable de animales.

Ley 26.842 (1997). Ley General de Salud.

Main, D. (2007). Interacción humano Animal. Conceptos en Bienestar Animal. World Animal Protection, Bristol.

Ministerio de Medio Ambiente (2017). Tercera Encuesta Nacional de Medio Ambiente. Ministerio de Medio Ambiente, Santiago de Chile.

Ministerio de Salud (2006). II Encuesta de calidad de vida y salud. Ministerio de Salud, Santiago de Chile.

Ministerio de Salud de Perú (2003). Guía sanitaria sobre Tenencia Responsable de Animales de Compañía: documento de trabajo para educación primaria. Ministerio de Salud de Perú, Lima.

Ministerio de Vivienda y Urbanismo (2015). Encuesta de Calidad de vida urbana. MINVU, Santiago de Chile.

Muldoon, J., Williams, J., Lawrence, A., Lakestani, N., y Currie, C. (2009). Promoting a Duty of Care Towards Animals Among Children and Young People. A literature review and findings from initial research to inform the development of interventions. Departament for Environment. London.

Organización Mundial de Sanidad Animal (2016). Código sanitario para los animales terrestres. OIE, París.

Soto, A. (2013). Análisis de un problema público no abordado, el caso de los perros vagabundos y callejeros en Chile. Magíster, Universidad de Chile.
Steurer, J. (2011). The Delphi method: an efficient procedure to generate knowledge. Skeletal radiology, 40(8):959-961.

Tetzlaff, J. M., Moher, D., y Chan, A.-W. (2012). Developing a guideline for clinical trial protocol content: Delphi consensus survey. Trials, 13(1):176.

Varela-Ruiz, M., Díaz-Bravo, L., y García-Durán, R. (2012). Descripción y usos del método Delphi en investigaciones del área de la salud. Investigación en educación médica, 1(2):90-95.

Young, E. y Quinn, L. (2002). Writing Effective Public Policy Papers: Guide for Policy Advisers in Central and Eastern Europe. Local Government and Public Service Reform Initiative. 